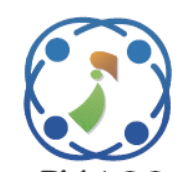

\title{
Mobile-based Routes Network Analysis for Emergency Response Using an Enhanced Dijkstra's Algorithm and AHP
}

\author{
Sayed Ahmed ${ }^{1 *}$ \\ Romani Farid Ibrahim ${ }^{2}$ \\ Hesham Ahmed Hefny ${ }^{3}$ \\ ${ }^{I}$ National Authority for Remote Sensing and Space Sciences, Egypt \\ ${ }^{2}$ Mohammed Bin Naïf Academy for Marine Science and Security Studies, Saudi Arabia \\ ${ }^{3}$ Department of Computer Sciences, Institute of Statistical Studies and Research, Cairo University, Egypt \\ * Corresponding author's Email: se.sayedahmed@gmail.com
}

\begin{abstract}
In emergencies cases, the main problem is how to arrive to an incident location in a minimum time. In such cases, using the classical distance based routing techniques does not always provide the optimal path in the time needed. In this paper, we present an enhanced routing technique which is based on Dijkstra's algorithm and Analytical Hierarchical Processing (AHP). Eight impendence factors that cause latency in emergency situations are analyzed using the AHP technique to give an overall weight for every road in Greater Cairo Region (GCR) road network. Then, the classical Dijkstra's algorithm applied on the weighted road network to give the best path between any two nodes in the network. The eight impedance factors considered are: Road Length, Travel Time, Road Type, Road Width, Velocity Limits, Traffic Volume, Mass Density, and Junction Delay. The suggested routing approach is implemented as a mobile application, and a comparison with the classical Dijkstra's algorithm is performed. The results show that the enhanced routing technique is more reliable than the classical one and more suitable for emergencies situations.
\end{abstract}

Keywords: AHP, GCR, Dijkstra's algorithm, Road network, Mobile application, Google Maps API.

\section{Introduction}

In an overcrowded area like Grater Cairo Region (GCR), Egypt, it is difficult to find the desired location, especially in emergency situations. So, it is mandatory to plan and invest wisely in infrastructure for sustainable urban transport due to the crucial role that transport plays in urban development by providing people with access to employment, healthcare, education, recreation, and other key services [1]. To find out a path between any two locations on a road network, either the path that has the minimum distance (the shortest path) or the path that has the minimum travel time (the best path) is to be selected. In emergency situations, the best path is preferred, as it takes the minimum time to reach an incident location which helps to save people life. Many factors affect the selection of a path such as road width, velocity limits, traffic volume, etc. These factors must be carefully evaluated and selected, as they can affect the total time of the arrival of the ambulance to the accident location, and a poor selection may cause delays in the arrival of the ambulance.

Nowadays, the Geographic Information System (GIS) technology is considered as one of the hottest research tools in the world that is regarded as a crucial in monitoring and emergency management [2]. A Geographic Information System is a computerized system that is designed to capture, store, manipulate, analyze, manage, visualize, and present spatial data in a way that a user can easily understand [3]. The shortest path between two nodes " $\mathrm{s}$ " and " $\mathrm{t}$ " in a network can be defined as the directed simple path from node "s" to node " $t$ " with the property that no other path has a lower weight [4]. Most applications solve the shortest path problem using the distance as a weight, but in emergencies, the other factors must be taken into account in order to minimize delays as much as 
possible. To assign paths that are suitable for emergency routing, we have to consider the other factors that affect the route selection process.

AHP, developed by Saaty (1980), is a Multi Criteria Decision Making (MCDM) technique that is used in complex decision-making problems. It adopts a multi-level hierarchical structure with a final goal and the alternatives to reach this goal [5]. Not only AHP helps the decision makers in prescribing a correct decision, but also it helps them to find the one that best fit for their needs and their understanding to the problem [6]. It is widely used in a variety of decision making situations in fields such as government, industry, healthcare, education, and business [6].

In this research, we have used the AHP method to analyze and compare the eight impedance factors that affect the selection of the best route, and give a weight (priority) for each factor. Then, we have assigned a general weight value for each route segment in the road network of the study area. Then, the classical Dijkstra's algorithm was enhanced by using this general weight value instead of the original weight value of the algorithm. Furthermore, the enhanced Dijkstra's algorithm was tested on a real world transport network (GCR, Egypt). The results obtained showed that the AHP weights values were consistent as its $C . R$. $=0.058$, i.e. $<0.1$, which means that the results obtained were reliable. Also, the results of applying the enhanced Dijkstra's algorithm on study area road network showed that it is very efficient, especially in emergencies cases.

The rest of this paper is organized as follows; Section 2 describes the state of the art literature, Section 3 describes the proposed methodology of the research, Section 4 describes the experimental setups and resulted obtained from this study, and finally Section 5 concludes the study and provides an outlook towards the future works.

\section{Related works}

In [1], an enhanced GIS-based network analysis was performed and applied on the Greater Cairo road network. The focus was on finding the best route between two locations on the road network and finding the nearest healthcare service providers to an incident location based on the travel time. The proposed method has integrated historical traffic data to be used in the analysis. The authors have used The Dijkstra's routing algorithm built into the ArcGIS Network Analyst Extension for implementing the network analysis. The proposed method has improved the travel time with $20 \%$ to $22 \%$, depending on the travel distances.
The authors did not consider the other factors that impose a delay in travelling through the road network.

In [2], the authors have utilized the capabilities of GIS in network analysis and visualization to support the decision making process in route selection to the nearest hospital by mapping the service area depending on the travel time. The authors did not consider any other factors that affect the travel time and did not consider the real-time traffic information on the road network.

In [4], the authors proposed an enhanced version of the Dijkstra's shortest path algorithm. In this version, the starting node is varying during the searching process. They have integrated the stack structure to maintain starting node, in order not to revisit the nodes. This enhancement has speeded up the searching process to practically. They have considered only the length of the path in their analysis and did not consider factors affecting travel through the network.

In [5], the authors have found that there are seven impedance factors that cause travel delay and have used the AHP technique to compare these factors and obtain the overall weight of each factor. The route with least travel time was obtained by applying Dijkstra's algorithm on the weighted road network. The results obtained from this emergency routing system have shown that the optimal path obtained by the modified routing method takes less time to reach the destination than the traditional routing method. The authors used the overall weight for the distance factor only to update the weight value of the Dijkstra's algorithm, so the result obtained depended only the distance of the route which does not provide accurate results.

In [7], the authors have used the ArcGIS software and Dijkstra's algorithm to solve the problem of finding the shortest path to reach a specialized hospital in Aurangabad city, Maharashtra State, India. The calculations of the shortest path were based on road distances; traffic congestion and state of the roads were not considered.

In [8], the authors developed a healthcare emergency response system based on GIS to obtain the best route from an incident location to the nearest healthcare service provider. They have considered the path with the least length as the best route. They did not consider any factors other than the path length. Their system enables end users to have access to all different tasks through a webbased interface. 
In [9], the authors have built an emergency response system based on GIS and Dijkstra's algorithm. Also, the system has integrated web services, and Asynchronous JavaScript and XML (Ajax) technologies to a web-based interface for getting the best routes from the specialized response team stations locations to an incident location. Their proposed system provided the optimal route depending only on the length of the route without considering any other factors that impose the delay on the travelling time.

In [10], the authors have developed a desktopbased emergency response management system based on GIS in Delhi, India. In this system, a detailed transportation network was maintained and integrated with real-time traffic data provided by NAVTEQ in India, which was used in analyzing the best routes to an incident location. Various analyses were performed in this system using GIS capabilities, such as network analysis, OriginDestination (OD) cost matrix, proximity analysis, and buffer analysis.

In [11], the authors have built a fire emergency response system based on GIS in Kumasi Metropolis, Ghana, to enable the Ghana National Fire Service (GNFS) to identify the best route from its location to any fire location. The best route was modelled based on the following factors only: travel distance, travel time, the slope of the roads, and the delays in travel times. No traffic information was provided.

In [12], the authors have proposed a decision support tool to model the routes network traffic congestion and provide alternative routes during rush hours in emergency situations. The system predicts the traffic flow and barriers during peak hours and suggests the alternative routes to reach hospitals at the time of emergency. They have used ArcGIS 9.3 network analyst tool and Dijkstra's algorithm to implement the shortest path analysis.

In [13], the authors proposed enhancement on Dijkstra's algorithm to overcome its two shortcomings (walking distance and ignoring number of transfers) by developing a penalty function that adds a small penalty cost to the cost of the path in case of stated shortcomings. Thus, the number of transfers and the number of repeated walking were minimized by slightly increasing the distance of the proposed path in the new route selection method. The results of the enhanced algorithm outperformed the traditional algorithm in terms of the average number of transfers and the average walking distance, but the enhanced algorithm had the worst running time.
In [14], the authors proposed a best route selection method by integrating Dijkstra's algorithm with Floyd-Warshall algorithm. Floyd-Warshall algorithm was used to determine the distance to the closest hospital, and then the fastest travel time route to this hospital was determined using Dijkstra's algorithm. Factors taken into account were the distance to the nearest hospital and the traffic flow density. In case of the occurrence of some problems on the way to the nearest hospital due to unexpected factors such as traffic incidents, the system moves the location of the hospital to other nearby hospital. Integrating Floyd-Warshall algorithm with Dijkstra's algorithm masked the defeats of each algorithm and speeded up the search time by reducing unnecessary decision makings.

In [15], the authors proposed an AHP driven multi-criteria decision making to find optimal path in real urban road network. They have used the classical Dijkstra's algorithm to select the shortest paths between any two stop points, then using the AHP method to select the optimal path among them. The authors used the AHP method to analyze, compare, and weight some factors that affect the selection of the optimal path. The factors considered were: distance, time, traffic volume, road width, the number of intersections, parking, and road's encroachment.

\section{The proposed method}

AHP method was used to analyze and compare eight impedance factors and calculate the overall priority of each factor through building comparison matrix. Then the classical Dijkstra's algorithm was enhanced by modifying its weight factor to represent the total weight for each road in the road network, and applied on a weighted road network to give the best route between any two given points. The proposed routing technique was implemented as a mobile application. The methodology of the proposed routing technique is shown in Fig. 1.

\subsection{Build AHP hierarchy}

The first step in AHP technique is to build the AHP hierarchy, i.e., representing the problem as a hierarchy in which the overall goal is the root and the set of criteria are branching from that root. In [5], the authors have found that there are seven impedance factors that cause travel delay. These factors are: Distance, Road Width, Road Type, Traffic Count, Population, Junction Delay, and Velocity. 


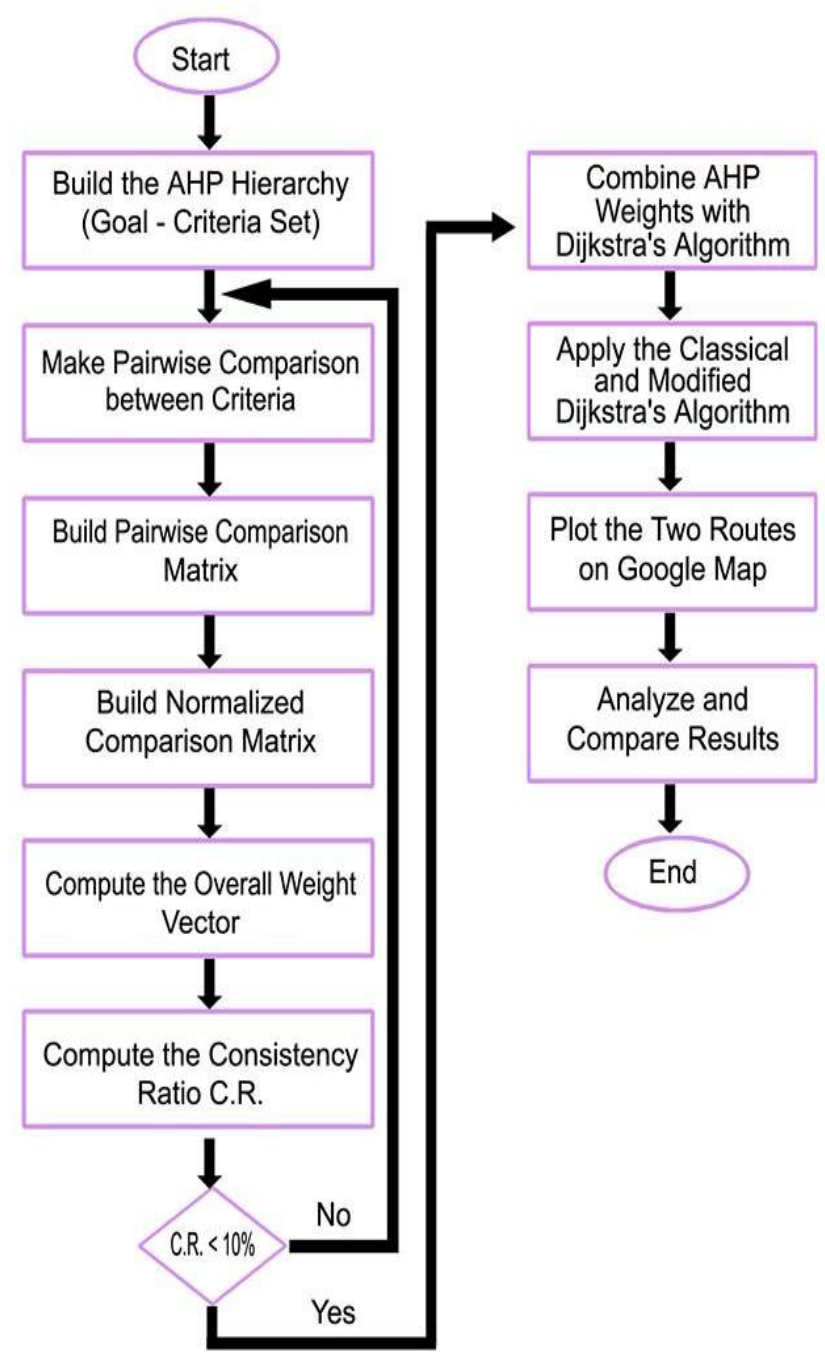

Figure. 1 The proposed methodology

There are several techniques to select such factors. In this research, the impedance factors were selected by investigating the real time traffic conditions and surveying traffic [5]. In our methodology, we have selected eight impedance factors that affect the selection of the best routes. The eight impedance factors considered to achieve the goal are represented here as a set of criteria. The impedance factors and the AHP hierarchy are shown in Fig. 2.

\subsection{Make pairwise comparisons}

In AHP process, the pairwise comparison has two roles. First, it is made to judge the importance of an alternative over another. Second, it is made to obtain the overall weight vector for all alternatives. Judging the importance of one alternative over another is a subjective issue that depends on one's perception. Table 1 lists the quantitative scales for the pairwise comparisons as developed by Saaty [16].

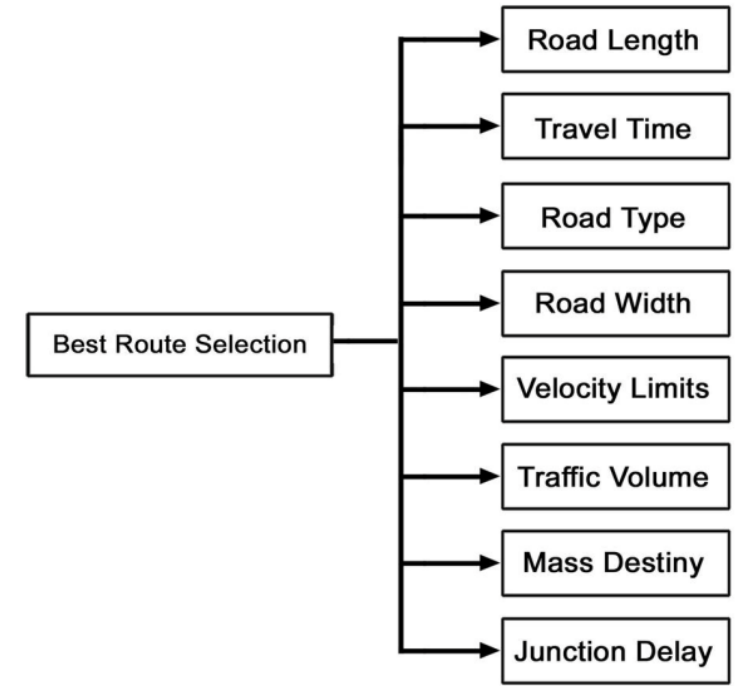

Figure. 2 The AHP hierarchy model of emergency routing

Table 1. Scales in pair wise comparisons

\begin{tabular}{|c|c|}
\hline Importance Scale & Definition \\
\hline 1 & Equally Importance \\
\hline 3 & Moderate Importance \\
\hline 5 & Strong Importance \\
\hline 7 & Extreme Importance \\
\hline 9 & Extremely More Importance \\
\hline $2,4,6,8$ & $\begin{array}{c}\text { Intermediate values between } \\
\text { Adjacent Scale Values }\end{array}$ \\
\hline
\end{tabular}

Depending on the number of criteria, the number of times that the pairwise comparison will be made is determined according to Eq. (1) [16].

$$
\text { No. of Comparisons }=n \times(n-1) / 2
$$

Such that:

$$
n=\text { the number of criteria }
$$

Therefore, in the proposed methodology, the number of pair wise comparisons is $8 \times(8-1) / 2$, i.e. 28 comparison.

\subsection{Build pairwise comparison matrix}

The output of the pairwise comparison made in the previous step is a comparison matrix which gives the importance ratios for each pair of alternatives [17]. The Comparison Matrix is an $\mathrm{n} x \mathrm{n}$ matrix, such that $\mathrm{n}$ is the number of criteria (alternative). 
The values in the comparison matrix are all positive. The main diagonal of this matrix is always 1 , which represent the value of comparing each criterion to itself. The values above the main diagonal can be expressed as $a_{i j}$, which indicates the importance of $i^{\text {th }}$ criterion over $j^{\text {th }}$ criterion. The values below the main diagonal are the inverse of the values above the main diagonal and can be expressed as $a_{j i}$ and can be calculated as $1 / a_{i j}$.

\subsection{Build the normalized comparison matrix}

The overall weight of each factor is calculated by normalizing the comparison matrix of the previous step using the geometric mean method given in Eq. (2) [16].

$$
r_{i}=\prod_{i=1}^{n}\left(a_{i j}\right)^{1 / n}
$$

Where $a_{i j}(i, j=1 \ldots n)$ are the comparison values in the pairwise comparison matrix and $n$ is number of alternatives.

\subsection{Compute the overall weight vector}

After computing the normalized comparison matrix, the overall weight vector can be calculated as shown in Eq. (3) [16].

$$
w_{i}=r_{i} / \sum_{j} r_{j}
$$

\subsection{Compute the consistency ratio C.R.}

The last step in AHP is to compute the consistency ratio of the pairwise comparison matrix. Consistency Ratio (C.R.) is a measure of the consistency of the comparison matrix according to your judge and perception.

The Consistency Ratio can be calculated as shown in Eq. (4) [5]. and Eq. (5) [5].

$$
\text { C.R. }=\text { C.I / R.I }
$$

Such that:

$$
\begin{aligned}
& \text { C.I. = Consistency Index } \\
& \text { R.I. = Random Index. }
\end{aligned}
$$

Saaty gave C.I. as a deviation or degree of consistency, and can be calculated as shown in Eq. (5) [5].

$$
\text { C.I. }=\frac{(\lambda \max -n)}{n-1}
$$

Such that:

$$
\begin{gathered}
\boldsymbol{\lambda}_{\max }=\text { the maximum Eigen value of comparison } \\
\text { matrix }
\end{gathered}
$$

$$
n=\text { the number of alternatives }
$$

Saaty proposed that we use this index by comparing it with the appropriate one. The appropriate consistency index is called Random Consistency Index (R.I.). The Random Consistency Index (R.I) is shown in Table 2 [16].

\subsection{Check the comparison matrix consistency}

If C.R. $<10 \%$, then the pairwise comparison matrix is consistent, and in this case the final decision matrix and the overall weight vector were produced, otherwise, we have to repeat the pairwise comparison, i.e., repeat the methodology steps from 3.2 to 3.7 [16]. The final decision matrix and the overall weight vector are shown in Table 3 and Table 4 respectively. The factors in these tables were selected as mentioned in section 3.1.

Table 2. Values of the random consistency index (RI) for small problems

\begin{tabular}{|c|c|}
\hline $\mathbf{n}$ & R.I. \\
\hline 1 & 0 \\
\hline 2 & 0 \\
\hline 3 & 0.58 \\
\hline 4 & 0.90 \\
\hline 5 & 1.12 \\
\hline 6 & 1.24 \\
\hline 7 & 1.32 \\
\hline 8 & 1.41 \\
\hline
\end{tabular}

Table 3. The final decision matrix

\begin{tabular}{|c|c|c|c|c|c|c|c|c|}
\hline & RL & TT & RT & RW & VL & TV & MD & JD \\
\hline RL & 1 & 0.11 & 0.5 & 0.14 & 0.11 & 0.11 & 0.11 & 2.0 \\
\hline TT & 9.0 & 1 & 9.0 & 3.0 & 2.0 & 1.0 & 1.0 & 9.0 \\
\hline RT & 2.0 & 0.11 & 1 & 1.0 & 0.14 & 0.11 & 0.11 & 3.0 \\
\hline RW & 7.0 & 0.33 & 1.0 & 1 & 0.5 & 1.0 & 1.0 & 7.0 \\
\hline VL & 9.0 & 0.5 & 7.0 & 2.0 & 1 & 1.0 & 1.0 & 7.0 \\
\hline TV & 9.0 & 1.0 & 9.0 & 1.0 & 1.0 & 1 & 1.0 & 7.0 \\
\hline MD & 9.0 & 1.0 & 9.0 & 1.0 & 1.0 & 1.0 & 1 & 7.0 \\
\hline JD & 0.5 & 0.11 & 0.33 & 0.14 & 0.14 & 0.14 & 0.14 & 1 \\
\hline
\end{tabular}

Table 4. The overall weight vector

\begin{tabular}{|c|c|c|}
\hline Criteria & Weight & Rank \\
\hline RL & $2.2 \%$ & 7 \\
\hline TT & $24.2 \%$ & 1 \\
\hline RT & $4.1 \%$ & 6 \\
\hline RW & $11.6 \%$ & 5 \\
\hline VL & $17.9 \%$ & 4 \\
\hline TV & $19.0 \%$ & 2 \\
\hline MD & $19.0 \%$ & 2 \\
\hline JD & $2.0 \%$ & 8 \\
\hline
\end{tabular}




\subsection{Combine AHP with Dijkstra's algorithm}

After generating the final weight vector, we will use it to compute an overall weight for each route in the routes network, and use this general weight to modify the weight factor of the classical Dijkstra's algorithm. Due to the diversity of measurement units for the impedance factors, it is impossible to express these in one factor, so a normalization process for each impedance factor was applied. The objective of the normalization process is to eliminate the units of measurement for the impedance factors and generate values between 0 and 1 , which can be easily combined in a single value. Not all impedance factors will be normalized in the same way. Some of them need to be maximized to select the best route, such as Velocity Limits, Road Type and Road Width. The other impedance factors need to be minimized to select the best route, such as Road Length, Initial Travel Time, Traffic Volume, Mass Destiny and Junction Delay. For those impedance factors that need to be maximized, the normalization formula will be as shown in Eq. (6).

$$
N F_{n}=1-\left(F_{n}-\operatorname{Min}\left(F_{n}\right) / \operatorname{Max}\left(F_{n}\right)-\operatorname{Min}\left(F_{n}\right)\right)
$$

Such that:

$$
\begin{aligned}
& N F_{n}=\text { Normalized Factor } F_{n} \\
& F_{n}=\text { Old value of Factor } F_{n} \\
& \text { Min }\left(F_{n}\right)=\text { Minimum value of the Factor } F_{n} \\
& \text { Max }\left(F_{n}\right)=\text { Maximum value of the Factor } F_{n} \\
& n=1 \ldots 8 .
\end{aligned}
$$

For those impedance factors that need to be minimized, the normalization formula will be as shown in Eq. (7).

$$
N F_{n}=\left(F_{n}-\operatorname{Min}\left(F_{n}\right) / \operatorname{Max}\left(F_{n}\right)-\operatorname{Min}\left(F_{n}\right)\right)
$$

Finally, the total weight for each route in the routes network will be calculated as shown in Eq. (8).

$$
W_{T}=\sum_{n=1}^{8}\left(N F_{n} \times W_{n}\right)
$$

Such that:

$W_{T}=$ the total weight for each route in the routes network in terms of the eight impedance factors.

$N F_{n}=$ Normalized Factor $F_{n}$.

$W_{n}=$ the corresponding weight of the factor $F_{n}$ in the overall weight vector.
After the general weight for each route has been calculated, it will be used as the weight value for the classical Dijkstra's Algorithm.

\subsection{Apply classical and modified Dijkstra's algorithm}

The classical and modified Dijkstra's algorithms are then being applied to the routes network to obtain both of the shortest route and the best route between the specified source and destination points using an Android Mobile Application.

\subsection{Plot the resulting routes on Google map}

The routes resulted from applying the classical and modified Dijkstra's algorithm to the routes network will then be plotted on a Google Map. Google provides Google Maps as web-based mapping services that provide a slick, highly responsive visual interface built based on AJAX technologies [18]. Google provides developers with free APIs that allow the integration of Google Map in their applications [19]. Google Map is acting as a base map for plotting the resulting routes. It is also used to translate the latitude and longitude of the source and the destination points into an exact physical address.

\subsection{Analyze and compare results}

The last step in the methodology is to compare the classical Dijkstra's algorithm result and the modified Dijkstra's algorithm result regarding the total distance and the total travel time in both cases.

\section{Results and discussion}

For testing the proposed routing technique in a near real-time GIS usage, experiments and comparisons were implemented to ensure that the proposed routing technique works properly.

\subsection{Input}

Study Area: The study area is the Greater Cairo metropolitan area. It is extended from $29^{\circ} 55^{\prime} 0^{\prime \prime} \mathrm{N}$ to $30^{\circ} 11^{\prime} 10^{\prime \prime} \mathrm{N}$ and from $30^{\circ} 20^{\prime} 50^{\prime \prime} \mathrm{E}$ to $31^{\circ} 27^{\prime}$ $50^{\prime \prime} \mathrm{E}$. Greater Cairo is the largest metropolitan area in Egypt, and the largest urban area in Africa [20]. It is the third largest urban area in the Islamic World after Jakarta and Karachi, and the world's $16^{\text {th }}$ largest metropolitan area [21]. It consists of Cairo Governorate, New Cairo City, Giza, Shubra ElKheima, $6^{\text {th }}$ of October City and Obour City, with a total population estimated at $20,500,000$ (as of 2012); area: $1,709 \mathrm{~km}^{2}$; density: $10,400 / \mathrm{km}^{2}$ [22]. 
For implementing and testing this system, a routes network of 3970 nodes and 11140 routes was stored in a SQLite database accompanying an android mobile application.

\subsection{AHP processing}

One of the contributions of this paper is that $C$. $R .=5.8 \%$, i.e., $<10 \%$. This consistency ratio was produced after 5 iterations of rebuilding the pairwise comparison matrix. Also the final Principle Eigenvalue computed in this methodology is $\boldsymbol{\lambda}_{\max }=$ 8.566 and the Eigenvector solution resulted after 5 iterations.

\subsection{Apply classical and modified Dijkstra's algorithm}

Based on an Android mobile application, we have implemented both the classical and modified Dijkstra's algorithms on the routes network of GCR. The main window of the application is shown in Fig. 3.

The start and end points are selected using the two drop-down lists, and their physical addresses are displayed in the textbox next to each dropdown list. These points are added to the Google Map using the "Add Locations" function in the upper right corner menu. Then, by using the "Find Routes" function, the classical and modified Dijkstra's algorithms are implemented, and the resulting routes are plotted on the map.

Fig. 4 shows the classical Dijkstra's algorithm result (the shortest route) in blue, and the modified Dijkstra's algorithm result (the best route) in magenta, between the start point 0 (red marker) and the end point 6 (green marker) along with a detailed comparison between the two results regarding distance, travel time, and speed.

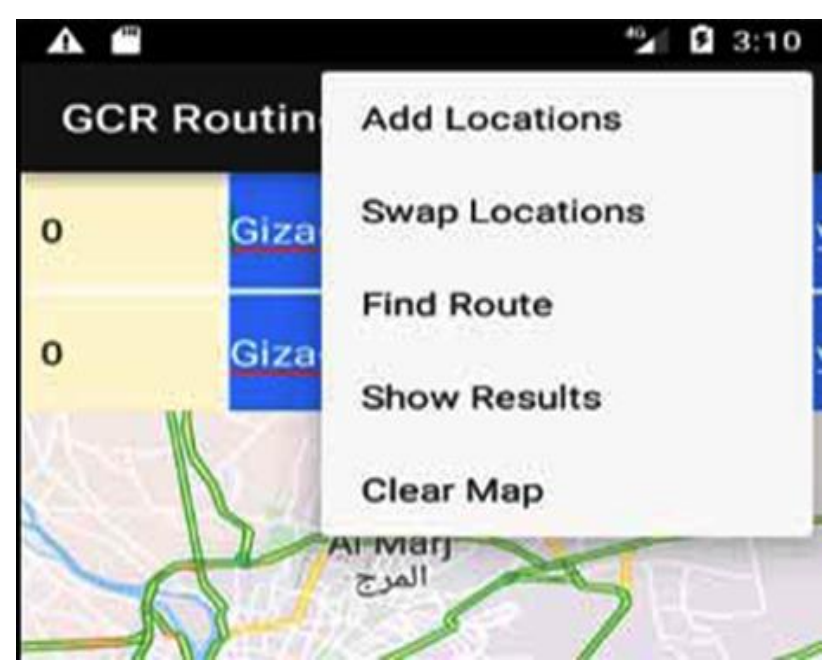

Figure. 3 The application main window
Fig. 5 shows the classical Dijkstra's algorithm result and the modified Dijkstra's algorithm result between the start point 1 (red marker) and the end point 3 (green marker) along with a detailed comparison between the two results. Also, Fig. 6 shows the classical Dijkstra's algorithm result and the modified Dijkstra's algorithm result between the start point 6 (red marker) and the end point 3969 (green marker) along with a detailed comparison between the two results. The final results are demonstrated in Table 5.

To ensure the efficiency of our proposed technique, we have randomly selected 2100 startend pairs from a total of 3970 stop points in the GCR road network. We have tested the classical and the modified Dijkstra's algorithm on the randomly selected pairs. The final results showed that the modified Dijkstra's algorithm outperforms its classical counterpart in terms of the average travel time and the average speed.

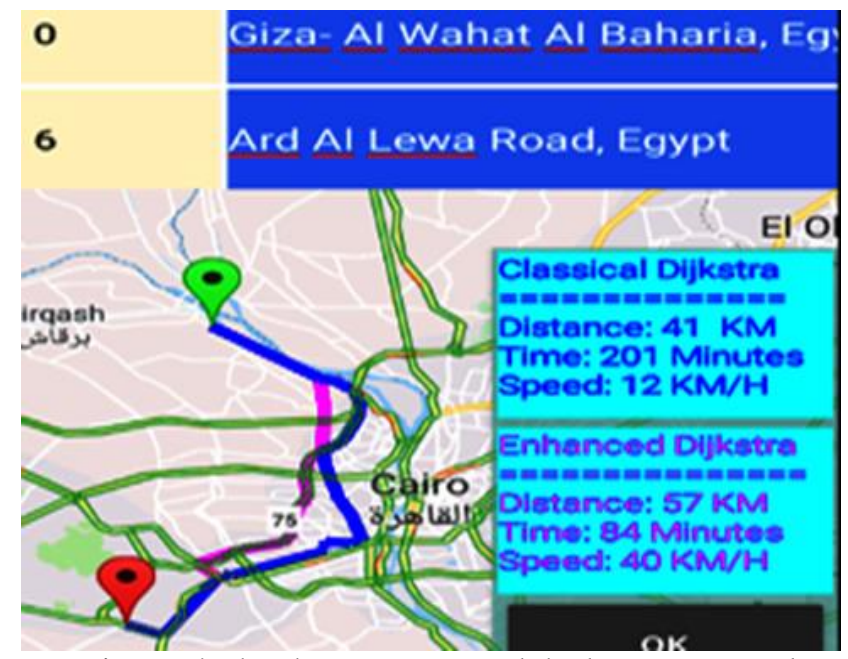

Figure. 4 The shortest route and the best route results between points 0 and 6 respectively

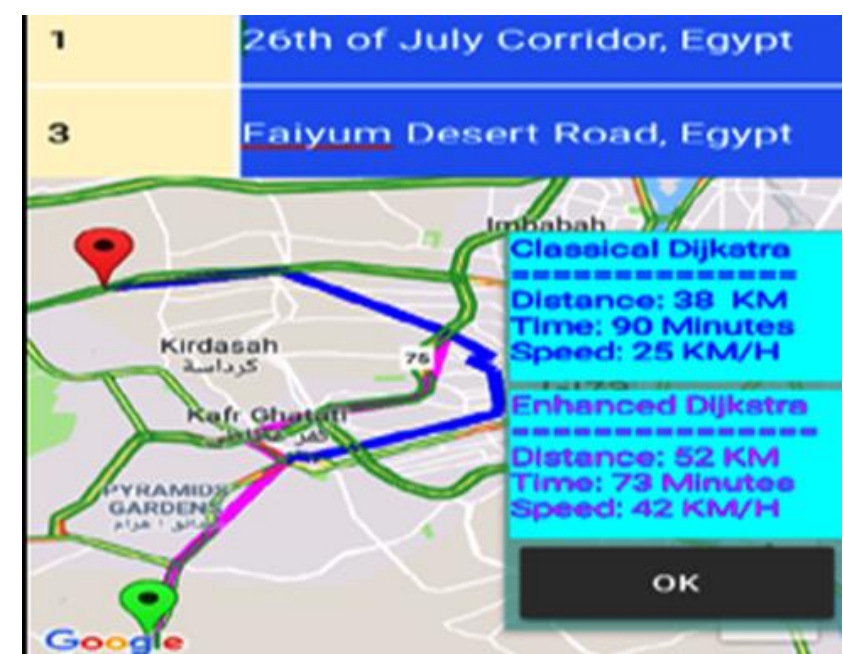

Figure. 5 The shortest route and the best route results between points 1 and 3 respectively 


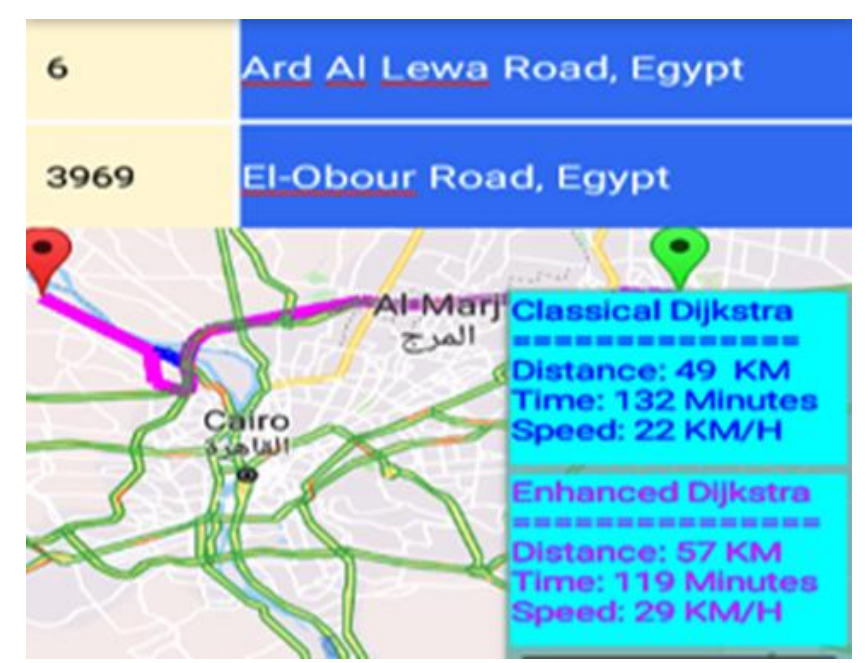

Figure. 6 The shortest route and the best route results for points 6 and 3969 respectively

Table 5. The final results

\begin{tabular}{|c|c|c|}
\hline Start - End & Classical Dijkstra & Modified Dijkstra \\
\hline \multirow{3}{*}{$0-6$} & Distance: $41 \mathrm{KM}$ & Distance: $57 \mathrm{KM}$ \\
\hline & $\begin{array}{c}\text { Travel Time: } 201 \\
\text { Min }\end{array}$ & $\begin{array}{c}\text { Travel Time: } 84 \\
\text { Min }\end{array}$ \\
\hline & Speed: $12 \mathrm{KM} / \mathrm{H}$ & Speed: $40 \mathrm{KM} / \mathrm{H}$ \\
\hline \multirow{3}{*}{$1-3$} & Distance: $38 \mathrm{KM}$ & Distance: 52 KM \\
\hline & $\begin{array}{c}\text { Travel Time: } 90 \\
\text { Min } \\
\end{array}$ & $\begin{array}{c}\text { Travel Time: } 73 \\
\text { Min } \\
\end{array}$ \\
\hline & Speed: $25 \mathrm{KM} / \mathrm{H}$ & Speed: 42 KM/H \\
\hline \multirow{3}{*}{$6-3969$} & Distance: $49 \mathrm{KM}$ & Distance: $57 \mathrm{KM}$ \\
\hline & $\begin{array}{c}\text { Travel Time: } 132 \\
\text { Min } \\
\end{array}$ & $\begin{array}{c}\text { Travel Time: } 119 \\
\text { Min } \\
\end{array}$ \\
\hline & Speed: $22 \mathrm{KM} / \mathrm{H}$ & Speed: $29 \mathrm{KM} / \mathrm{H}$ \\
\hline \multirow{3}{*}{$\begin{array}{l}\text { Avg. of } 2100 \\
\text { Random } \\
\text { Start \& End }\end{array}$} & Distance: $11 \mathrm{KM}$ & Distance: $23 \mathrm{KM}$ \\
\hline & $\begin{array}{c}\text { Travel Time: } 161 \\
\text { Min }\end{array}$ & $\begin{array}{l}\text { Travel Time: } 119 \\
\text { Min }\end{array}$ \\
\hline & Speed: 4 KM/H & Speed: $10 \mathrm{KM} / \mathrm{H}$ \\
\hline
\end{tabular}

The results comparison indicates that the enhanced routing technique is most suitable for emergency cases and for daily traffic routing.

By comparing our proposed technique's results with Dijkstra's algorithm, the results obtained from the AHP technique are more reliable as the obtained C.R. $=0.058$ which is much less than 0.1. Compared to other researchers, we have used 8 impedance factors rather than using 7 impedance factors as in [5] and 6 impedance factors as in [15].

\section{Conclusion and future work}

In this paper, an enhanced routing technique based on Dijkstra's algorithm and the AHP method was implemented on an Android mobile application and applied to the Greater Cairo routs network.
We have improved the classical Dijkstra's algorithm by substituting its weight factor with an overall weight factor form the AHP method. In addition, we have tested the modified algorithm and compared it with the classical Dijkstra's algorithm.

Also, we used the Google Maps as base maps to draw the resulting routes and to convert the endpoints of locations to a physical addresses.

The results obtained show that the proposed technique is more efficient than the classical Dijkstra's algorithm. Because the average travel time was enhanced by $26 \%$ and the average speed was enhanced by $60 \%$.

In our future work, we will investigate more factors that affect the selection of the best route, such as the safest routes, the economic routes, the routes with the less accidents rates.

Comparing different short path algorithms that can be applied in emergencies, such as $\mathrm{A}^{*}$ algorithm, will be investigated.

Furthermore, implementation of our proposed technique in cloud computing environment as a cloud application, to decrease its response time and to be more flexible.

\section{Acknowledgment}

The authors would like to thank Ihab Samir, Marwa Moustafa, and Amira Sobhy for their great efforts and support in this research.

\section{References}

[1] S. Ahmed, R. F. Ibrahim, and H. A. Hefny, "GIS-Based Network Analysis for the Roads Network of the Greater Cairo Area", In: Proc. of 2nd International Conference on Applied Research in Computer Science and Engineering, 2017.

[2] A. E. Abdel Aziz, R. F. Abdalla, N. A. Gism, A. E. Mohammed, and S. E. Khidir, "Route Network Analysis in Khartoum City", Journal of Engineering and Computer Science, Vol. 17, No. 1, pp.50-57, 2016.

[3] V. Shashikiran, T. S. Kumar, N. S. Kumar, V. Venkateswaran, and S. Balaji, "Dynamic road traffic management based on krushkal's algorithm", In: Proc. of International Conference on Recent Trends in Information Technology, pp.200-204, 2011.

[4] D. Kong, Y. Liang, X. Ma, and L. Zhang, "Improvement and Realization of Dijkstra Algorithm in GIS of Depot", In: Proc. of International Conference in Control, 
Automation and Systems Engineering, pp.1-4, 2011.

[5] V. Mali, M. Rao, and S. S. Mantha, "AHP Driven GIS Based Emergency Routing in Disaster Management", In: Proc. of Communications in Computer and Information Science, pp.237-248, 2013.

[6] S. Yang and C. Li, "An enhanced routing method with Dijkstra algorithm and AHP analysis in GIS-based emergency plan", In Proc. of 18th International Conference on Geoinformatics, pp.1-6, 2010.

[7] A. Dabhade, K. V. Kale, and Y. Gedam, "Network Analysis for Finding Shortest Path in Hospital Information System", International Journal of Advanced Research in Computer Science and Software Engineering, Vol.5, No.7, pp.618-623, 2015.

[8] A. Gubara, A. Amasha, Z. Ahmed, and S. El Ghazali, "Decision Support System Network Analysis for Emergency Applications", In: Proc. of 9th International Conference on Informatics and Systems, pp.40-44, 2014.

[9] N. Kai, Z. Y. Ting, and M. Y. peng, "Shortest Path Analysis Based on Dijkstra's Algorithm in Emergency Response System", TELKOMNIKA Indonesian Journal of Electrical Engineering, Vol.12, No.5, pp.3476-3482, 2014.

[10] A. Bhagat and N. Sharma, "GIS-Based Application for Emergency Preparedness and Management Accelerating Response System through GIS", In: Proc. of the 14th Esri India User Conference, pp.1-7, 2013.

[11] E. K. Forkuo and J. A. Quaye-Ballard, "GIS Based Fire Emergency Response System", International Journal of Remote Sensing and GIS, Vol.2, No.1, pp.32-40, 2013.

[12] S. Naithani, A. Choudhry, and S. Chauhan, "Decision Support System for Emergency Response", European Scientific Journal, Vol.2, No.3, pp.680-687, 2013.

[13] A. Bozyigit, G. Alankus, and E. Nasiboglu, "Public Transport Route Planning: Modified Dijkstra's Algorithm", In: Proc. of the 2nd International Conference on Computer Science and Engineering, pp.502-505, 2017.

[14] Risald, A. E. Mirino, and Suyoto, "Best Routes Selection Using Dijkstra and Floyd-Warshall Algorithm", In: Proc. of International Conference on Information \& Communication Technology and System, pp.155-158, 2017.

[15] S. Kukadapwar and D. Parbat, "Estimation of Optimal Path on Urban Road Networks Using AHP Algorithm", International Journal for
Traffic and Transport Engineering, Vol.6, No.1, pp.13-24, 2016.

[16] T. L. Saaty, "The Analytic Hierarchy Process", McGraw-Hill International, New York, 1980.

[17] V. Mali, M. Rao, and S. S. Mantha, "Enhanced routing in disaster management based on GIS", In: Proc. of International Conference on Intuitive Systems and Solutions, pp.14-18, 2012.

[18] S. Ahmed, M. Badawy, E. Zidan, R. F. Ibrahim, "Implementation of an Investment Information System Based on Google Maps API", International Journal of Advanced Research in Computer and Communication Engineering, Vol. 4, No. 9, pp.383-388, 2015.

[19] P. Aleksandar, S. Pletl, and B. Pejic, "An expert system for tourists using Google Maps API", In: Proc. of the 7th International Symposium on Intelligent Systems and Informatics, pp.317-322, 2009.

[20] Demographia World Urban Areas \& Population Projections (PDF), Demographia, April 2009, retrieved 9 July 2009.

[21] R. L. Forstall, R.P. Greene, and J.B. Pick, "Which are the largest? Why published populations for major world urban areas vary so greatly", In: Proc. of City Futures Conference, University of Illinois at Chicago, pp.34, 2004.

[22] The Evolving Urban Form: Cairo, New Geography, 13 June 2012, retrieved 9 Oct 2015. 